\title{
Psychometric evaluation of a screening question for persistent depressive disorder
}

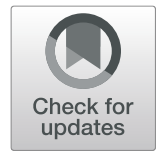

\author{
Elisa Brinkmann * (D), Sarah Glanert, Michael Hüppe, Ana Sofia Moncada Garay, Sophie Tschepe, \\ Ulrich Schweiger and Jan Philipp Klein
}

\begin{abstract}
Background: About one in five patients with depression experiences a chronic course. Despite the great burden associated with this disease, there is no current screening instrument for Persistent Depressive Disorder (PDD). In the present study, we examine a short screening test, the persistent depression screener (PDS), that we developed for DSM-5 PDD. The PDS is comprised of one question that is administered following an initial self-assessment for depression.
\end{abstract}

Methods: Ninety patients from an inpatient clinic/day clinic specialized in treating depression completed the PDS. They were also assessed using a structured clinical interview covering the DSM-5 criteria for PDD. Retest reliability was examined after two weeks ( $n=69,77 \%)$.

Results: In this sample, the prevalence of PDD was $64 \%$. Sensitivity of the PDS was $85 \%$ with a positive predictive value of $80 \%$. Specificity was $63 \%$. Positive and negative likelihood ratios were 2.3 and .24 , respectively. Agreement between the PDS results and the outcome of the clinical interview was moderate (Cohen's Kappa $\mathrm{K}=.48$ ([95\%-Cl .28, .68], $p<.001$, $S E=0.10)$ ). Prevalence-adjusted bias-adjusted Kappa was PABAK $=.53$. Retest reliability of the PDS was moderate (Cohen's Kappa $\mathrm{K}=.52([95 \%-\mathrm{Cl} .3, .74], \mathrm{p}<.001, \mathrm{SE}=0.11))$.

Conclusions: The present study shows that the PDS - when applied following a self-rating depression scale - might be a valid and reliable way to detect PDD. However, the results of the PDS must be confirmed by a diagnostic interview.

Keywords: Persistent depressive disorder, Dysthymia, Depressive disorders, Screening question, Rater agreement, Diagnostic interview

\section{Background}

Worldwide, depression is one of the leading causes of burden of disease [1]. Mortality of people currently affected by depression is considerably higher compared to people not suffering from depression [2]. Lifetime prevalence for a major depressive episode is assumed to be around 17\% [3-5]. About one fifth of people suffering from major depression experience relevant symptoms for two years or longer and therefore meet the criteria for persistent depressive disorder (PDD; [6-10]). The longer a person suffers from a depressive disorder, the less likely recovery becomes [11]. In the fifth version of the Diagnostical and Statistical Manual of Mental Disorders (DSM), the American Psychiatric Association

\footnotetext{
* Correspondence: elisabrinkmann@gmail.com

Department of Psychiatry and Psychotherapy, University of Lübeck, 23562 Lübeck, Germany
}

(APA) summarized various forms of chronic depression in the section "Persistent Depressive Disorder" (DSM-5; [12]). Compared to patients with episodic forms of depression, patients with PDD have a higher rate of comorbidities and even suicide attempts $[3,9,13]$.

The identification and subsequent treatment of depressive disorders, especially chronic forms, is essential as they cause intense suffering for those affected, their families and society as a whole [14]. The U.S. Preventive Service Task Force and its Canadian counterpart recommend screenings for depression provided that adequate treatment is available $[15,16]$. Tried and tested screening instruments are available for depressive disorders (e.g. [17]). Very short screening instruments have proven to adequately detect depression, like the Patient Health Questionnaire-2 (PHQ-2; $[18,19])$ or the 5-Item World Health Organization Well-Being Index (WHO-5; [20]). 
Other screening instruments specifically developed for depression were successfully tested in particular sub groups such as pregnant and postpartum women. The U.S. Preventive Service Task Force states that there is a moderate net health benefit in screening this specific population [16]. It is necessary to identify chronic courses of depression since treatment of chronically depressed patients seems to be more successful when their particular needs and deficits, such as interpersonal problems and comorbidity with personality disorders, are directly addressed [21]. Patients with chronic depression seem to respond better to specific forms of therapy, e.g. the cognitive behavioral analysis system of psychotherapy (CBASP), than to unspecified forms of therapy $[22,23]$.

To our knowledge, no screening for PDD has been developed so far. After protracted forms of depression had been conceptualized as dysthymia in DSM-III, a question that screened for this condition was developed and tested [24]. However, in DSM-III dysthymia is somewhat differently defined than PDD in DSM-5. In particular, PDD can be diagnosed if depressive symptoms have been present almost all of the time (persistent depressive episode). This underlines the need and urgency for an updated screening.

In the present study we examine the persistent depression screener (PDS) - a screening question for DSM-5 Persistent Depressive Disorder - that can be administered following a self-rating scale for depression. The question reads: "The previous questions covered various symptoms of depression. Now, please consider: When was the last period of two months or longer that you were not impaired by these symptoms?". We hypothesize that the PDS has adequate psychometric properties to detect PDD when accompanied by an initial self-assessment of depressive symptomatology. Specifically, we hypothesize that PDS results will at least moderately agree with results of a structured diagnostic interview.

\section{Methods \\ Participants}

Participants were recruited at the inpatient/day clinic treatment program for depression at the Department of Psychiatry and Psychotherapy, University of Lübeck, Germany. Participants did not receive financial compensation. The present study uses data from the ICARE-Study (Investigating Care Dependency And its Relation to out$\operatorname{com} E$ ) designed to investigate the German version of the Care Dependency Questionnaire [25]. The ICARE-study was conducted in accordance with the Declaration of Helsinki and it was approved by the ethics committee of the University of Lübeck. Inclusion and exclusion criteria for the study were modeled on the treatment program's admission criteria. The treatment program focusses on psychotherapy for depression (mainly CBASP [26] and
MCT [27]) and lasts for six weeks. Minimum age for participation in the study was 18 years. An adequate understanding of the German language and informed written consent were required. As we aimed to only include subjects who were not yet familiar with the treatment program, we only accepted patients to the study if it was their first admission to the treatment program. Exclusion criteria were acute suicidality, a history of schizophrenia, delusional disorder, substance use disorder or bipolar disorder as well as a known diagnosis of an acute somatic illness that requires treatment. Only data from patients who completed both the PDS and the clinical interview was analyzed.

\section{Materials}

\section{Persistent depression screener (PDS)}

We developed the PDS, a paper-and-pencil screening composed of one question. It was administered following a self-rating instrument for depressive symptoms: the Quick Inventory of Depressive Symptomatology (QIDS-SR; [17, 28]). The PDS is based on the DSM-5 criteria for PDD and focusses on criterion $\mathrm{C}$ for chronicity of the symptoms ("During the 2-year period of the disturbance, the individual has never been without symptoms ... for more than two months at a time"). The translated screening question reads:

"The previous questions covered various symptoms of depression. Now, please consider: When was the last period of two months or longer that you were not impaired by these symptoms?"

The following response options were given:

a) less than a year ago.

b) more than a year but less than 2 years ago.

c) more than 2 years but less than 5 years ago.

d) more than 5 years but less than 10 years ago.

e) more than 5 years ago.

Answers a) and b) were determined to be indicative of a likely absence of PDD ("PDS negative"). Answers c) to e) indicate a likely presence of PDD ("PDS positive").

Before we collected the data for the main sample, we conducted a pilot study $(N=5)$ to ensure comprehensibility and feasibility of the PDS. Participants of the pilot study completed the PDS and the interview and these results were examined. The screening outcomes of two participants differed from their interview-based diagnoses. We conducted additional semi-structured interviews with these patients to determine their interpretation of the screening question. Based on this information, we slightly amended the wording of the PDS to improve clarity. The modified and the original question were then presented to the two participants. Both expressed a clear preference for the modified question. As a result, we 
used the revised PDS to collect data for the main sample. Participants of the pilot study were excluded from all further statistical analyses.

\section{Clinical interview for PDD}

Trained raters collected diagnostic information on the presence and course of the depressive disorder according to DSM-5 criteria for depressive disorders using a structured interview $[29,30]$. The interview was based on the Structured Clinical Interview for DSM (SCID). The order of the questions was changed to increase ease of administration in the diagnosis of PDD (assessment for current depressive episode, past depressive episode and persistent depressive episode; assessment of number of depressive episodes; assessment for presence of dysthymic syndrome; assessment of early versus late onset). Other studies also successfully employed this interview (e.g. [31, 32]). Participants were diagnosed with PDD when meeting DSM-5 criteria for a pure dysthymic syndrome, for a persistent major depressive episode, for persistent depressive disorder with intermittent major depressive episodes, with current episode as well as without current episode. The clinical interview served as the criterion standard for the PDS in this paper.

\section{Quick inventory of depressive symptomatology - self report (QIDS-SR)}

The German translation of the Quick Inventory of Depressive Symptomatology - Self Report was applied prior to the PDS to establish if the patient suffered from depressive symptoms. It is a valid and reliable self-assessment tool of depression severity [28]. It consists of 16 questions concerning depressive symptoms experienced in the last 7 days. The total score ranges from 0 to 27 with higher scores reflecting a greater severity of symptoms [17].

\section{Hamilton-Rating-Depression-Scale-6 (HRDS-6)}

The Hamilton-Rating-Depression-Scale-6 is a short (six items) clinician-rated assessment scale for the severity of depressive symptomatology. It is the shortened version of the original scale with 17 items [33]. Symptoms are rated based on the patient's report and the clinician's observation with total scores ranging from 0 to 22 [34].

\section{Procedure}

Between May 2017 and April 2018 all patients were contacted and informed about the study within the first days of their admission to the treatment program. If patients were eligible for the study (e.g. no discontinuation of treatment, for further information please refer to Fig. 1), they were briefed in detail on the goal and the procedure of the study and had to provide informed consent to participate. Subsequently trained graduate students with a clinical psychology major (EB and ST) conducted the clinical interview and the HRDS-6. We handed out several questionnaires covering demographic information, the QIDS-SR and the PDS to participants within the first week of their treatment. Patients were included in the analysis sample if the questionnaires as well as the interview were completed. Questionnaire data was anonymized using the examiner's initials followed by a serial number (e.g. EB034). After 2 weeks, patients completed another self-assessment of depressive symptoms and the PDS was handed out again to collect data for

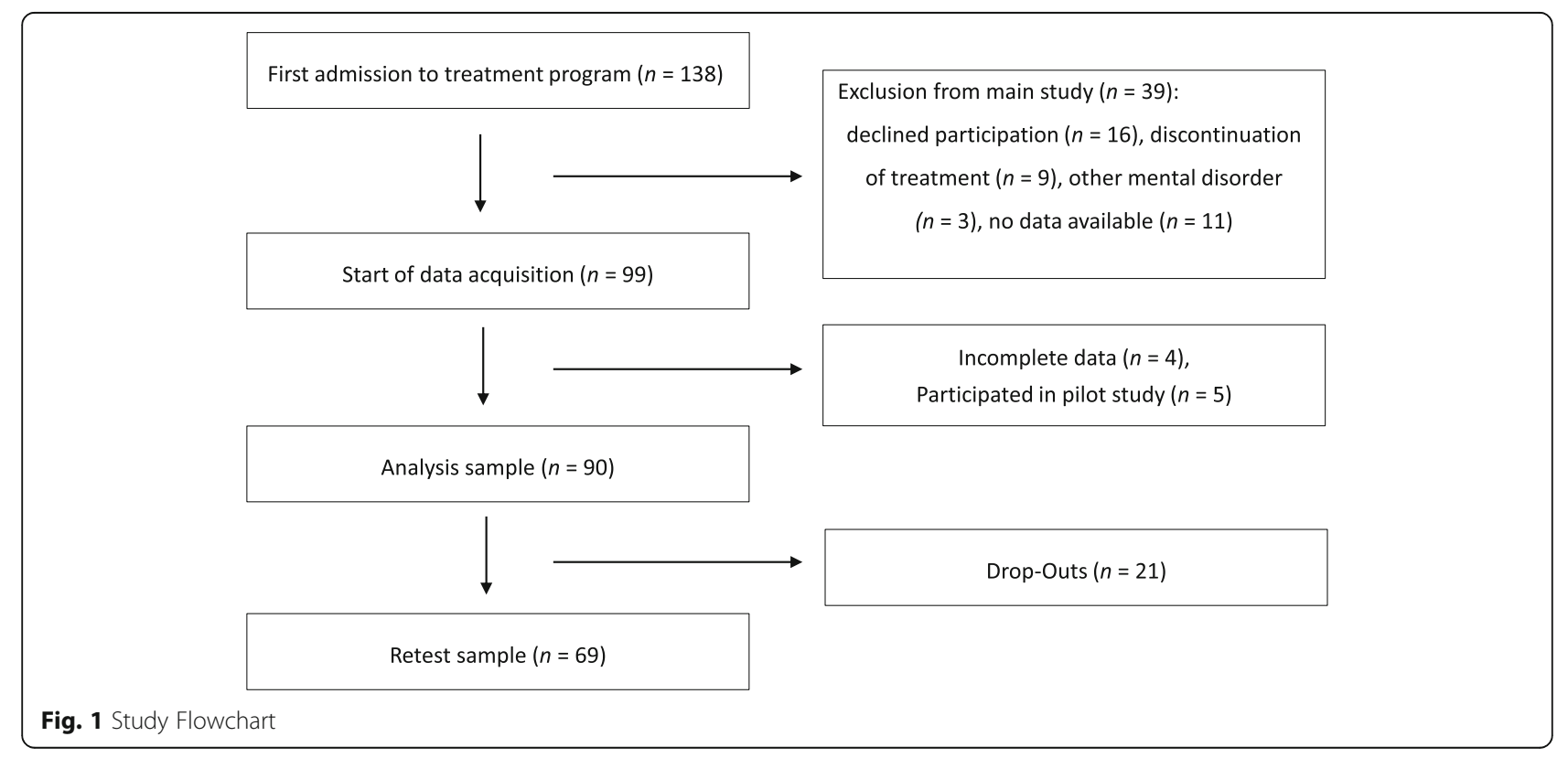


retest reliability. For detailed information on the procedure of the study, please refer to Fig. 1.

\section{Statistical analyses}

Statistical analyses were conducted using SPSS (IBM SPSS Statistics for Windows, version 22.0). All statistical tests were two-tailed tests with significance levels set at $p \leq .05$. Standard errors $(S E)$ and $95 \%$-confidence intervals $(C I)$ are provided in the result section. Missing values were not substituted. To assess the quality of the PDS in a clinical context, we report common measures like sensitivity, specificity, predictive values as well as likelihood ratios. It should be noted that unlike sensitivity, specificity and likelihood ratios, predictive values depend on the prevalence of PDD in the sample [35].

To examine agreement between the PDS result and the diagnosis derived from the clinical interview, Cohen's Kappa (к) was utilized. $\mathrm{k}$ is a coefficient for rater agreement taking into account chance agreement. Values for $\kappa$ range between -1 and +1 , with +1 indicating total agreement between outcomes [36]. Landis and Koch's (1977) guideline describes agreement as poor at a value of 0 , as slight when $\mathrm{\kappa}=0-.20$, as fair when $\mathrm{\kappa}=.21-.40$, as moderate when $\mathrm{\kappa}=.41-.60$, as substantial when $\mathrm{\kappa}=.61-.80$ and as almost perfect when $\mathrm{K}=.81-1$ [36]. However, Kraemer et al. (2012) state that with DSM-5-diagnoses in a clinical context $\mathrm{k}$-values ranging between .41 and .60 are realistic and values between .21 and .40 are acceptable [37].

As a high prevalence of PDD diagnoses was expected in our sample, an alternative calculation of $\mathrm{k}$ was conducted. The prevalence-adjusted bias-adjusted Kappa $(P A B A K)$ takes into account the categorization by the PDS and the prevalence of the disease. A bias index (BI) is calculated to check for possibly differing proportions of PDD diagnoses deriving from the clinical interview and the PDS. If the marginal proportions of outcomes are equal, there is no bias between PDS and interview $(B I=0)$. The $B I$ reaches a maximum of 1 , when there is no overlap in the instruments' ratings. A prevalence index (PI) is reported to assess the potentially differing relative probability of the two categories likely diagnosis of $P D D$ and unlikely diagnosis of PDD. PI is 0 , if both categories are equally likely. If only one of the two categories occurs in this sample, $P I$ is $+/-1$. A very high probability of one category increases chance agreement between the outcomes of the PDS and the clinical interview. Higher values of chance agreement result in lower $\mathrm{K}$ values. For further information about the calculation of $P A B A K$, please refer to Byrt, Bishop and Carlin (1993) [38].

Additionally, Cramér's $V(5 \times 2$ table $)$ is reported as a measure of association between the result of the original (not dichotomized) screening question and the outcome of the corresponding interview question. Different thresholds of the screening answers were also examined to assess the most beneficial proportion of sensitivity and specificity with the Youden-Index J (sensitivity + specificity -1 ): the higher the value of $J$, the more reliable the test outcome [39].

\section{Results}

\section{Characteristics of the sample}

The analysis sample comprised 90 individuals. For detailed information on participant recruitment, please refer to the study flowchart (Fig. 1). Out of the 90 participants, $18(20 \%)$ were inpatients and $72(80 \%)$ were day clinic patients. Table 1 shows participants' demographic and clinical characteristics.

\section{Psychometric properties of the PDS}

The diagnosis based on the clinical interview concurred with the PDS result in 69 cases $(N=90,77 \%)$ as Table 2 illustrates. As shown in Table 2, sensitivity of the PDS was $85 \%$, with $80 \%$ accurate positive screening outcomes (positive predictive value). Specificity was $63 \%$. The resulting Youden-Index was $J=.48$. There were $16 \%$ false-negative and $38 \%$ false-positive results. Of the patients with a negative PDS, $69 \%$ did in fact not have a diagnosis of PDD (negative predictive value). The resulting positive likelihood ratio was 2.3, meaning it was 2.3 times more likely that a subject with PDD had a positive PDS than subjects without PDD having a positive PDS. The negative likelihood ratio was .24, meaning it was 4.2 times more likely that a subject without PDD had a negative PDS compared to subjects with PDD having a negative PDS. Jaeschke et al. (1994) regard values of these magnitudes as small, but sometimes important [40].

Cohen's $\mathrm{k}$ was .48 ([95\%-CI .28, .68], $p<.001, S E=0.10)$. The strength of agreement can hence be considered moderate with a range from fair to substantial [41]. Bias between PDS results and clinical interviews was negligible for the value of $\kappa(B I=.03)$. The prevalence effect was moderate $(P I=.32)$. This moderate prevalence effect implies that Cohen's $\mathrm{K}$ might be an underestimation of the agreement between the PDS and the clinical interview. We therefore calculated the prevalence-adjusted bias-adjusted Kappa (PABAK), which was .53. Accordingly, this can be interpreted as a moderate agreement between the PDS results and the outcomes of the clinical interviews [41]. When the answers to the PDS were not dichotomized but treated as an ordinal variable for agreement with the interview results, a significant and strong relation of Cramér's $V=.59, p<.001$ was determined. In this sample 98\% ( $n=88)$ of participants suffered from a depressive disorder, the remaining two patients who were not diagnosed with depression were correctly categorized by the PDS as not likely suffering from PDD. 
Table 1 Demographics and clinical characteristics of the analysis sample $(N=90)$

\begin{tabular}{|c|c|}
\hline Demographics & $n(\%)$ \\
\hline Sex (female) & $46(51)$ \\
\hline Married and cohabiting & $24(27)$ \\
\hline Higher secondary school qualifying for university & $30(33)$ \\
\hline Employed & $51(57)$ \\
\hline \multirow[t]{2}{*}{ Day clinic patients } & $72(80)$ \\
\hline & mean (SD / range) \\
\hline Age (years) & $41.67(13.70 / 19-64)$ \\
\hline \multicolumn{2}{|l|}{ Use of health care resources in past 12 months } \\
\hline nights spent as an inpatient in a clinical facility & $12.41(24.05)$ \\
\hline visits to a psychiatrist/neurologist & $3.82(5.67)$ \\
\hline visits to a psychotherapist & $6.35(9.97)$ \\
\hline Diagnoses & $n(\%)$ \\
\hline PDD & $58(64)$ \\
\hline persistent depressive episode & $17(19)$ \\
\hline $\begin{array}{l}\text { with intermittent depressive episodes, with } \\
\text { current episode }\end{array}$ & $39(43)$ \\
\hline $\begin{array}{l}\text { with intermittent depressive episodes without } \\
\text { current episode }\end{array}$ & $1(1)$ \\
\hline with pure dysthymic syndrome & $1(1)$ \\
\hline Recurrent depressive disorder & $27(30)$ \\
\hline with current depressive episode & $23(26)$ \\
\hline currently in partial remission & $4(4)$ \\
\hline First depressive episode & $3(3)$ \\
\hline Other & $2(2)$ \\
\hline severe anxiety disorder & $1(1)$ \\
\hline recent suicide attempt & $1(1)$ \\
\hline Up to five depressive episodes in their lifetime & $58(64)$ \\
\hline \multirow[t]{2}{*}{ Early onset of depression (before age of 21) } & $50(56)$ \\
\hline & mean (SD) \\
\hline Number of depressive episodes & $8.93(15.83)$ \\
\hline \multicolumn{2}{|l|}{ Depression severity } \\
\hline HRDS-6 & $10.58(4.44) *$ \\
\hline QIDS-SR & $15.14(5.77) *$ \\
\hline
\end{tabular}

Note. SD standard deviation. * The average severity of depression can be rated as moderate

When a patient had been suffering from depressive symptoms for more than 2 years, the PDS categorized the patient as having a likely diagnosis of PDD (answers [c] to [e]). The threshold for a PDD diagnosis can be shifted to examine its accuracy. Table 3 shows that when examining the Youden-Index there are two possible thresholds in the answers to the PDS - the original one at more than 2 years (answers [c] to [e]) and the threshold at more than 5 years (answers [d] and [e]). The latter provided a larger Youden-Index of $J=.56$ compared to
Table 2 Agreement between the diagnoses from the interview and the PDS

\begin{tabular}{lllll}
\hline & & \multicolumn{2}{l}{$\begin{array}{l}\text { PDD diagnosis based on } \\
\text { the clinical interview }\end{array}$} & \\
\cline { 3 - 4 } & & present & not present & \\
\hline likely PDD diagnosis & present & $49(85 \%)$ & $12(38 \%)$ & $61(68 \%)$ \\
based on PDS result & not present & $9(16 \%)$ & $20(63 \%)$ & $29(32 \%)$ \\
& total & 58 & 32 & 90 \\
\hline
\end{tabular}

Note. PDS persistent depression screener. PDD Persistent depressive disorder. The table shows the agreement (number of subjects, \%) between the diagnoses from the clinical interview and the PDS results about chronicity of the depressive symptomatology

$J=.48$ of the original threshold. However, it could only offer a sensitivity of 59\%, which does not meet the requirements of how a screening instrument for depression should perform [42]. It can be concluded that the original threshold at more than 2 years (answers [c] to [e]) showed the highest agreement coefficient in combination with a high sensitivity and a reasonable specificity. It offered the most accurate and valuable information.

To examine the understanding of the PDS, we tested for differences in the agreement between outcomes of the interview and outcomes of the PDS by controlling for level of education. We found a slightly better value for Cohen's $\mathrm{\kappa}$ for patients with a higher level of education $(\kappa=.51([95 \%$-CI $.20, .82], p<.005, S E=0.16, n=30)$ compared to patients with lower education $(\kappa=.46$ ([95\%-CI .22, .70], $p<.001, S E=0.12, n=59$ ). Both values can be interpreted as moderate [41]. Sensitivity and specificity of the PDS were 83 and $67 \%$ for patients with higher education, whereas sensitivity for patients with lower education was $85 \%$ and specificity was $60 \%$.

\section{Retest reliability of the PDS}

Data was collected again from 69 participants after an interval of 2 weeks to determine retest reliability (77\% of main analysis sample). Agreement between the first result of the PDS and its repetition was 80\% (55 of 69 cases). The agreement rate with Cohen's $\mathrm{k}=.52$ $([95 \%$-CI .3, .74], $p<.001, S E=0.11)$ can be interpreted as moderate [41]. After adjusting for a small bias $(B I=-.06)$ and a moderate prevalence effect $(P I=.39)$, the agreement rate was supported by $P A B A K=.59$. When the answers of the PDS are not dichotomized, but examined as an ordinal variable for agreement, a moderate to substantial relation was detected, Spearman's $\rho=.49, p<.01$.

\section{Discussion}

To our knowledge, the present study is the first to examine a screening question for PDD. We showed that a short screening of one item is sufficient to distinguish between cases of PDD and non-PDD when administered 
Table 3 Diagnostical thresholds of the PDS

\begin{tabular}{llllll}
\hline $\begin{array}{l}\text { Threshold for last period } \\
\text { without complaints }\end{array}$ & $k$ & $p$ & sensitivity & specificity & $\mathrm{J}$ \\
\hline $\begin{array}{l}\text { More than 1 year ago } \\
\text { (a vs. b-e) }\end{array}$ & .30 & .002 & $90 \%$ & $38 \%$ & .28 \\
$\begin{array}{l}\text { More than } 2 \text { years ago } \\
\text { (a-b vs. C-e) }\end{array}$ & .48 & $<.001$ & $85 \%$ & $63 \%$ & .48 \\
$\begin{array}{l}\text { More than } 5 \text { years ago } \\
\text { (a-c vs. d-e) }\end{array}$ & .48 & $<.001$ & $59 \%$ & $97 \%$ & .56 \\
$\begin{array}{l}\text { More than } 10 \text { years ago } \\
\text { (a-d vs. e) }\end{array}$ & .18 & .007 & $26 \%$ & $97 \%$ & .23 \\
\hline
\end{tabular}

Note. PDS persistent depression screener. The table shows the calculated value of Kappa (K) with the associated level of significance $(p)$, sensitivity, specificity and the resulting Youden-Index $(J)$ for varying diagnostical thresholds of the PDS

after a symptom severity rating for depression in a treatment program for depressive disorders. A good sensitivity and positive predictive value with a reasonable specificity suggest that in this very setting, i.e. an inpatient/day clinic treatment program for depression, the outcome of the PDS is a valid indicator of further diagnostic effort concerning the presence of PDD. The outcomes of the PDS moderately corresponded with the diagnoses stemming from clinical interviews. The range of the $\mathrm{K}$ value can be interpreted as fair to substantial [36]. As mentioned before other interpretations suggest that in clinical contexts this range is acceptable to realistic [37]. The prevalence-adjusted bias-adjusted Kappa $(P A B A K)$ value supports our findings. Therefore, we are satisfied with the results.

The agreement between the screening results and the repetition 2 weeks later was moderate, therefore retest reliability is assessed as good in this specific setting. The value of the corresponding $P A B A K$ supports this. Twenty-one (23\%) out of 90 patients in the main analysis sample differed in their outcomes of the PDS and the interview. The majority of these cases $(n=14,67 \%)$ chose a screening answer around the threshold of the diagnostic criterion for PDD (answers [b] or [c]). We believe that the specificity of $63 \%$ can be accepted for the PDS. If a patient is categorized as likely suffering from $P D D$ by the PDS, this result must be confirmed with a clinical diagnostic interview.

As this study was part of a bigger project, we did not define the sample size for the psychometric assessment of the PDS a priori. According to Sim and Wright (2005) a sample size of $n=43$ is acceptable for the detection of a coefficient of $\kappa=.50$ with a two-tailed test (Null Value of $\mathrm{K}=.00$ ) and a test power of $90 \%$ [43]. Our sample size $(N=90)$ is therefore adequate to test the main research hypothesis.

With the present instrument being the only screening for PDD, we cannot directly compare it to similar measures. The U.S. Preventive Services Task Force (2009) notes that most depression screenings show a sensitivity between 80 and $90 \%$ and a specificity of 70 to $85 \%$ [37]. The PDS does not reach that value for specificity but complies with the value for sensitivity. The previously described screening instrument for DSM-III-Dysthymia showed slightly better sensitivities (89-92\%) but lower specificities (35-62\%) in Mental Health settings [24].

The psychometric properties of the PDS compare favorably to the properties of existing depression screeners, namely the PHQ-2, the Hospital Anxiety and Depression Scale (HADS) and the WHO-5. The PHQ-2 is a short screening for depression, consisting of only two questions. Compared to the PDS, the PHQ-2 showed a slightly better sensitivity of $87 \%$ and better specificity of $78 \%$ at the chosen cut-off point for the diagnosis of major depression, but a less favorable value of $\kappa$ of .43 [19]. A series of studies on other screening instruments for depression including the HADS and the WHO-5 showed similar results for sensitivity but somewhat better specificity compared to the PDS [18, 44, 45]. We therefore consider the psychometric properties of our measures to be in an acceptable range.

The PDS was examined in a treatment program specialized in the treatment of depression, so prevalence of PDD was expected to be higher than in other clinical contexts. Predictive values were good but should be interpreted with caution. These measures are dependent on the prevalence of the examined disorder and therefore influenced by the high base rate of PDD diagnoses in this particular sample. The other measures are reliable nevertheless, because sensitivity and specificity as well as likelihood ratios are independent of prevalence. The impact of prevalence on $\mathrm{k}$ was considered and examined by the calculation of $P A B A K$.

\section{Strengths and limitations}

Strengths of the current study include that our sample is similar to a representative population sample reporting depressive symptoms in a number of demographic characteristics including age, gender, employment status and notably education (most samples of patients in psychotherapy programs are more highly educated than the general population) [46]. We did not find that education level substantially affected our screening results. Also, we performed a broad range of calculations to psychometrically test the PDS.

Regarding limitations of this study, it should be noted that patients were repeatedly asked about the chronicity of their depression both by clinical staff in the treatment program and research staff. The repeated administration of these questions might have influenced the patients' answers. Compared to patients treated in a setting less specialized in depression, patients in our study likely had more opportunities to reflect on when they have last felt 
free from symptoms of depression. This might have inflated the accuracy of our screening test. Finally, the pretest probability for a depressive disorder was comparatively high in this sample given that data was collected in a treatment program specialized in depression.

\section{Future research}

Future studies should examine the practicality of the PDS in different medical contexts with a lower prevalence of depression (e.g. in general psychiatric care). Future studies should also verify whether the screening question could be reworded for use in a clinical intake interview. In this setting clinicians could ask the following question after the assessment of current depressive symptomatology: "When have you last experienced a period of two months or longer when you were not impaired by depressive symptoms?" It has been shown that asking simple questions on depressed mood and anhedonia in a clinical assessment interview can perform similar to longer instruments [47].

\section{Conclusion}

The persistent depression screener (PDS) can be administered economically after an initial severity assessment in patients with depression. It showed good sensitivity and moderate accuracy in comparison to the results of a clinical interview. Its brevity, the limited administration effort and low cost make it an economic instrument to elicit information about chronicity of depression. However, the outcome of the PDS must be confirmed by a diagnostic interview. If our results hold up in future studies, mental health clinics could utilize this screening question to detect PDD and thus provide patients with specific treatment.

\section{Abbreviations \\ APA: American Psychiatric Association; CBASP: Cognitive Behavioral Analysis System of Psychotherapy; DSM: Diagnostical and Statistical Manual of Mental Disorders; HADS: Hospital Anxiety and Depression Scale; HRDS-6: Hamilton- Rating-Depression-Scale-6; ICARE: Investigating Care Dependency And its Relation to outcome; MCT: Metacognitive Therapy; PABAK: Prevalence- adjusted bias-adjusted Kappa; PDD: Persistent Depressive Disorder; PDS: Persistent depression screener; PHQ-2: Patient Health Questionnaire-2; QIDS-SR: Quick Inventory of Depressive Symptomatology - Self-Report; SCID: Structured Clinical Interview for DSM; WHO-5: 5-Item World Health Organization Well-Being Index}

\section{Acknowledgements}

The authors would like to thank all patients for their participation and the staff of the treatment program for their support in the conduct of this study.

\section{Funding}

Not applicable.

\section{Availability of data and materials}

Individual participant data can be shared with researchers who provide a methodologically sound proposal to JPK. Proposals may be submitted up to 36 months following publication of this paper.

\section{Authors' contributions}

EB, SG, ASMG and JPK designed the study and obtained ethical review board permission. Patients were recruited by EB, SG, ASMG, ST and JPK. EB conducted the statistical analyses. The results were interpreted, and the manuscript was written by EB with substantial input from JPK. All authors commented on the manuscript and approved the final version.

\section{Ethics approval and consent to participate}

This study was approved by the ethics committee of the University of Lübeck. All Participants received verbal and written descriptions of the study and provided their written informed consent.

\section{Consent for publication}

Not applicable.

\section{Competing interests}

The authors declare that they have no competing interests.

\section{Publisher's Note}

Springer Nature remains neutral with regard to jurisdictional claims in published maps and institutional affiliations.

Received: 13 August 2018 Accepted: 3 April 2019

Published online: 23 April 2019

References

1. Ferrari AJ, Charlson FJ, Norman RE, Patten SB, Freedman G, Murray CJL, et al. Burden of depressive disorders by country, sex, age, and year: findings from the global burden of disease study 2010. Hay PJ, editor. PLoS Med. 2013;10: e1001547.

2. Lasserre AM, Marti-Soler H, Strippoli M-PF, Vaucher J, Glaus J, Vandeleur $\mathrm{CL}$, et al. Clinical and course characteristics of depression and all-cause mortality: a prospective population-based study. J Affect Disord. 2016; 189:17-24.

3. Angst J, Gamma A, Rössler W, Ajdacic V, Klein DN. Long-term depression versus episodic major depression: results from the prospective Zurich study of a community sample. J Affect Disord. 2009;115:112-21.

4. Hasin DS, Goodwin RD, Stinson FS, Grant BF. Epidemiology of major depressive disorder: results from the National Epidemiologic Survey on alcoholism and related conditions. Arch Gen Psychiatry. 2005;62:1097-106.

5. Kessler RC, Berglund P, Demler O, Jin R, Koretz D, Merikangas KR, et al. The epidemiology of major depressive disorder: results from the National Comorbidity Survey Replication (NCS-R). J Am Med Assoc. 2003;289:3095-105.

6. Eaton WW, Shao H, Nestadt G, Lee BH, Bienvenu OJ, Zandi P. Populationbased study of first onset and chronicity in major depressive disorder. Arch Gen Psychiatry. 2008;65:513.

7. Gilmer WS, Trivedi MH, Rush AJ, Wisniewski SR, Luther J, Howland RH, et al. Factors associated with chronic depressive episodes: a preliminary report from the STAR-D project. Acta Psychiatr Scand. 2005;112:425-33.

8. Kennedy N, Abbott R, Paykel ES. Remission and recurrence of depression in the maintenance era: long-term outcome in a Cambridge cohort. Psychol Med. 2003:33:827-38.

9. Murphy JA, Byrne GJ. Prevalence and correlates of the proposed DSM-5 diagnosis of chronic depressive disorder. J Affect Disord. 2012;139:172-80.

10. Satyanarayana S, Cox B, Sareen J. Prevalence and correlates of chronic depression in the Canadian community health survey: mental health and well-being. J Psychiatry. 2009;54:389-98.

11. Keller MB, Lavori PW, Mueller TI, Endicott J, Coryell W, Hirschfeld RMA, et al. Time to recovery, chronicity, and levels of psychopathology in major depression: a 5-year prospective follow-up of 431 subjects. Arch Gen Psychiatry. 1992;49:809-16.

12. American Psychiatric Association. Diagnostical and statistical manual of mental disorders. 5th ed. Arlington: American Psychiatric Publishing; 2013.

13. Vandeleur CL, Fassassi S, Castelao E, Glaus J, Strippoli M-PF, Lasserre AM, et al. Prevalence and correlates of DSM-5 major depressive and related disorders in the community. Psychiatry Res. 2017;250:50-8.

14. Lépine J-P, Briley M. The increasing burden of depression. Neuropsychiatr Dis Treat. 2011;7:3-7.

15. MacMillan HL, Patterson CJS, Wathen CN, The Canadian Task Force on Preventive health care. Screening for depression in primary care: 
recommendation statement from the Canadian Task Force on Preventive Health Care. Can Med Assoc J. 2005;172:33-5.

16. Siu AL, Bibbins-Domingo K, Grossman DC, Baumann LC, Davidson KW, Ebell $M$, et al. Screening for depression in adults: US Preventive Services Task Force recommendation statement. JAMA. 2016;315:380-7.

17. Rush AJ, Trivedi MH, Ibrahim HM, Carmody TJ, Arnow B, Klein DN, et al. The 16-item quick inventory of depressive symptomatology (QIDS), clinician rating (QIDS-C), and self-report (QIDS-SR): a psychometric evaluation in patients with chronic major depression. Biol Psychiatry. 2003;54:573-83.

18. Arroll B, Goodyear-Smith F, Crengle S, Gunn J, Kerse N, Fishman T, et al. Validation of PHQ-2 and PHQ-9 to screen for major depression in the primary care population. Ann Fam Med. 2010;8:348-53.

19. Löwe B, Kroenke K, Gräfe K. Detecting and monitoring depression with a two-item questionnaire (PHQ-2). J Psychosom Res. 2005;58:163-71.

20. Topp CW, Østergaard SD, Søndergaard S, Bech P. The WHO-5 wellbeing index: a systematic review of the literature. Psychother Psychosom. 2015;84:167-76.

21. Jobst A, Brakemeier E-L, Buchheim A, Caspar F, Cuijpers P, Ebmeier KP, et al. European psychiatric association guidance on psychotherapy in chronic depression across Europe. Eur Psychiatry. 2016;33:18-36.

22. Schramm E, Kriston L, Zobel I, Bailer J, Wambach K, Backenstrass M, et al. Effect of disorder-specific vs nonspecific psychotherapy for chronic depression: a randomized clinical trial. JAMA Psychiatry. 2017:74:233.

23. Wiersma JE, Van Schaik DJF, Hoogendorn AW, Dekker JJ, Van HL, Schoevers RA, et al. The effectiveness of the cognitive behavioral analysis system of psychotherapy for chronic depression: a randomized controlled trial. Psychother Psychosom. 2014;83:263-9.

24. Burnam MA, Wells KB, Leake B, Landsverk J. Development of a brief screening instrument for detecting depressive disorders. Med Care. 1988:775-89.

25. Geurtzen N, Keijsers GP, Karremans JC, Hutschemaekers GJ. Patients' care dependency in mental health care: development of a self-report questionnaire and preliminary correlates. J Clin Psychol. 2018;74:1189-206. https://doi.org/10.1002/jclp.22574.

26. McCullough JP, Schramm E, Penberthy JK. CBASP as a distinctive treatment for persistent depressive disorder. London and New York: Routledge; 2015.

27. Wells A. Metacognitive therapy for anxiety and depression. New York: Guilford Press; 2009.

28. Roniger A, Späth C, Schweiger U, Klein JP. A psychometric evaluation of the German version of the quick inventory of depressive symptomatology (QIDS-SR16) in outpatients with depression. Fortschritte Neurol Psychiatr. 2015;83:17-22.

29. Klein JP, Belz M. Psychotherapie chronischer depression: Praxisleitfaden CBASP. Göttingen: Hogrefe; 2014.

30. Faßbinder $E$, Klein JP, Sipos $V$, Schweiger U. Therapie-Tools Depression. 1. Auflage. Weinheim Basel: Beltz; 2015.

31. Klein JP, Kensche M, Becker-Hingst N, Stahl J, Späth C, Mentler T, et al. Development and psychometric evaluation of the interactive test of interpersonal behavior (ITIB): a pilot study examining interpersonal deficits in chronic depression. Scand J Psychol. 2016;57:83-91.

32. Klein JP, Stahl J, Hüppe M, McCullough JP, Schramm E, Ortel D, Sondermann S, Schröder J, Moritz S, Schweiger U. Do interpersonal fears mediate the association between childhood maltreatment and interpersonal skills deficits? A matched cross-sectional analysis. Psychother Res. 2018. https://doi.org/10.1080/10503307.2018.1532125. Epub ahead of print.

33. Bech P, Gram LF, Dein E, Jacobsen O, Vitger J, Bolwig TG. Quantitative rating of depressive states. Acta Psychiatr Scand. 1975;51:161-70.

34. Bech P. Rating scales for mood disorders: applicability, consistency and construct validity. Acta Psychiatr Scand. 1988:78:45-55.

35. Lalkhen AG, McCluskey A. Clinical tests: sensitivity and specificity. Contin Educ Anaesth Crit Care Pain. 2008;8:221-3.

36. Cohen J. A coefficient of agreement for nominal scales. Educ Psychol Meas. 1960;20:37-46

37. Kraemer HC, Kupfer DJ, Clarke DE, Narrow WE, Regier DA. DSM-5: how reliable is reliable enough? Am J Psychiatry. 2012;169:13-5.

38. Byrt T, Bishop J, Carlin JB. Bias, prevalence and kappa. J Clin Epidemiol. 1993; 46:423-9.

39. Youden WJ. Index for rating diagnostic tests. Cancer. 1950;3:32-5.

40. Jaeschke R, Guyatt GH, Sackett DL, Guyatt G, Bass E, Brill-Edwards P, et al. Users' guides to the medical literature: III. How to use an article about a diagnostic test B. what are the results and will they help me in caring for my patients? J Am Med Assoc. 1994;271:703-7.

41. Landis JR, Koch GG. The measurement of observer agreement for categorical data. Biometrics. 1977;33:159-74

42. U.S. Preventive Services Task Force. Screening for depression in adults: U.S. Preventive Services Task Force recommendation statement. Ann Intern Med 2009;151:784-792.

43. Sim J, Wright CC. The kappa statistic in reliability studies: use, interpretation, and sample size requirements. Phys Ther. 2005;85:257-68.

44. tpdelLöwe B, Spitzer RL, Gräfe K, Kroenke K, Quenter A, Zipfel S, et al. Comparative validity of three screening questionnaires for DSM-IV depressive disorders and physicians' diagnoses. J Affect Disord. 2004;78: 131-40.

45. Saipanish R, Lotrakul M, Sumrithe S. Reliability and validity of the Thai version of the WHO-five well-being index in primary care patients. Psychiatry Clin Neurosci. 2009;63:141-6.

46. Späth C, Hapke U, Maske U, Schröder J, Moritz S, Berger T, et al. Characteristics of participants in a randomized trial of an internet intervention for depression (EVIDENT) in comparison to a national sample (DEGS1). Internet Interv. 2017;9:46-50.

47. Whooley MA, Avins AL, Miranda J, Browner WS. Case-finding instruments for depression. Two questions are as good as many. J Gen Intern Med. 1997;12: $439-45$
Ready to submit your research? Choose BMC and benefit from:

- fast, convenient online submission

- thorough peer review by experienced researchers in your field

- rapid publication on acceptance

- support for research data, including large and complex data types

- gold Open Access which fosters wider collaboration and increased citations

- maximum visibility for your research: over $100 \mathrm{M}$ website views per year

At BMC, research is always in progress.

Learn more biomedcentral.com/submissions 